\title{
Finding the Efficiency Status and Efficient Projection in Multiobjective Linear Fractional Programming: A Linear Programming Technique
}

\author{
S. Morteza Mirdehghan and Hassan Rostamzadeh \\ Department of Mathematics, College of Sciences, Shiraz University, Shiraz, Iran \\ Correspondence should be addressed to S. Morteza Mirdehghan; mirdehghan@shirazu.ac.ir
}

Received 30 December 2015; Accepted 8 August 2016

Academic Editor: Wlodzimierz Ogryczak

Copyright (C) 2016 S. M. Mirdehghan and H. Rostamzadeh. This is an open access article distributed under the Creative Commons Attribution License, which permits unrestricted use, distribution, and reproduction in any medium, provided the original work is properly cited.

\begin{abstract}
Multiobjective linear fractional programming (MOLFP) problems are the important problems with special structures in multiobjective optimization. In the MOLFP problems, the objective functions are linear fractional functions and the constraints are linear; that is, the feasible set is a polyhedron. In this paper, we suggest a method to identify the efficiency status of the feasible solutions of an MOLFP problem. By the proposed method, an efficient projection on the efficient space for an inefficient solution is obtained. The proposed problems are constructed in linear programming structures.
\end{abstract}

\section{Introduction}

Multiobjective programming (MOP) is a well-known research field in optimization and operations research. The multiobjective optimization problems have several objective functions and a set of feasible solutions. There are several special structures in multiobjective optimization problems. One class of them includes multiobjective linear fractional programming (MOLFP) problems which contain several linear fractional objective functions and a polyhedron as the feasible set.

There are many methods to find the efficient solutions of multiobjective optimization problems which are constructed based on iterative, scalarization, interactive, and other methods. If the objective functions or the constraints are not linear, then we should solve a mathematical programming problem which is not linear, but if the objective functions and constraints are linear, then the current approaches, usually, solve linear programming (LP) problems which are usually more desirable than nonlinear programming problems in the computationally efforts.

One of the well-known and the interesting methods to find the efficiency status of a feasible solution in MOP is Benson's method which was proposed by Benson [1]
This method receives an initial feasible solution of the MOP problem and identifies the efficiency status of that. If the feasible solution is not efficient, then this approach produces an efficient solution as a projection of the underassessment inefficient solution. Another well-known method to find the efficient solution of an MOP problem is the weighted sum method. By this method the MOP problem is converted to an optimization problem with a single objective function. The weights of the objective functions of the MOP problems are specified by the decision-maker's point of view. If the weights are nonnegative, then the optimal solutions are weakly efficient and in particular if the weights are positive, then the optimal solutions are efficient. Besides the weighted sum approach, the $\varepsilon$-constraint method is also a well-known technique to solve MOP problems. There is no aggregation of objective functions; instead only one of the original objectives is optimized, while the others are transformed to constraints. This approach was introduced by Haimes et al. [2], and an extensive discussion can be found in Chankong and Haimes [3].

The objective functions of the MOLFP problems are fractional but the numerator and denominator of the objective functions are affine. To check the efficiency status of a feasible solution of an MOLFP problem by the traditional approaches, 
we should solve a fractional programming problem which is not desirable compared to solving a linear programming problem.

A linear fractional programming (LFP) problem includes a polyhedron as the feasible set and a fractional objective function whose numerator and denominator are affine functions. In other words, the numerator and denominator of the objective function are convex and concave simultaneously, but the whole of the objective function usually is not convex and concave, while it is quasi convex and quasi concave, simultaneously. So all local optimal solutions of an LFP problem are global optimal solutions. Moreover, if the LFP problem has an optimal solution, then it has an extreme optimal solution. To guarantee the existence of an optimal solution of an LFP problem, we suppose that the feasible set is bounded and the denominator of the objective function is positive or negative in the whole of the feasible set. Charnes and Cooper [4] have shown that an LFP problem can be solved by a linear programming technique; that is, the optimal solution of the constructed linear programming problem is the optimal solution of the LFP problem, where the sign of the denominator of the objective function is not changed in the whole of the feasible set. There are some researches to focous on solving LFP problems such as Gilmore and Gomory [5], Martos [6], Illés et al. [7], Tantawy [8], and Odior [9].

Because of the importance of solving MOLFP problems in multiobjective optimization, some articles have been published on the subject of solving MOLFP problems. Most of the techniques in the published researches are based on interactive, iterative, linearization, parametric, and decomposition methods. Kornbluth and Steuer [10] found a weakly efficient solution of an MOLFP problem based on a simplex based algorithm. Meteve and Gueorguieva [11] identified a weakly efficient solution using a nonlinear programming problem. Also Caballero and Hernández [12] proposed a controlled estimation method to find a set of weakly efficient solutions. Dinkelbach [13] solved an MOLFP problem using a parametric technique and this approach was extended by Almogy and Levin [14], Crouzeix et al. [15], Falk and Palocsay [16], Tammer et al. [17], Skiscimi and Palocsay [18], and Schaible and Shi [19].

In the parametric technique of Tammer et al. [17], the parameters are calculated by solving some equations and the obtained feasible solution, necessarily, is not efficient solution. Most of parametric techniques extracted from Dinkelbach [13] cannot guarantee solving the MOLFP problems. Also some of approaches to solve MOLFP problems are based on iterative techniques such as Costa [20], Kuno [21], Phuong and Tuy [22], Dai et al. [23], Costa and Alves [24], Matejaš and Perić [25], and Valipour et al. [26]. Most of techniques for solving MOLFP problems try to find an efficient solution for an MOLFP problem.

In this paper, at first, we propose an approach which identifies the efficiency status of an arbitrary feasible solution of an MOLFP problem. Then, we propose an approach which not only identifies the efficiency status of an arbitrary feasible solution but also finds an efficient projection of an arbitrary feasible solution. In these two approaches, we construct linear programming problems regarding the MOLFP problem. Moreover, we show that in evaluating the under-assessment feasible solution by the proposed linear programming problem, in the first approach, if the optimal value is zero, then the under-assessment feasible solution is an efficient solution. In the second approach, the optimal solution of the linear programming problem is a weakly efficient solution. In particular, if the optimal solution of the proposed problem is unique, then the optimal solution is an efficient solution. The behavior and application of our proposed problems are similar to Benson's [1] problem and $\mathcal{\varepsilon}$-constraint problem, proposed by Haimes et al. [2], for solving MOLFP problems. The differences among Benson's method, $\varepsilon$-constraint method, and our proposed methods are the structures of the suggested problems. Our proposed problems are linear programming problems, while Benson's and the $\varepsilon$-constraint problem, corresponding to an MOLFP problem, not only have nonlinear objective functions but also have nonlinear constraints.

In Section 2, we introduce some notions, definitions, and properties which are required in the main discussions. In Section 3, the main discussions and properties are presented. Two numerical examples to illustrate our approaches are presented in Section 4, and the final section is conclusion.

\section{Preliminaries}

2.1. Linear Fractional Programming Problem. The linear fractional programming problems are modeled as follows:

$$
\begin{array}{ll}
\text { Min } & f(x)=\frac{c x+\alpha}{d x+\beta}, \\
\text { s.t. } & x \in X=\left\{x \in R^{n}: A x \leq b, x \geq 0\right\},
\end{array}
$$

where $X$ is a nonempty and bounded set, $b \in \mathbb{R}^{m}$ and $c, d \in$ $\mathbb{R}^{n}$ are vectors of (known) coefficients, $A \in \mathbb{R}^{m \times n}$ is a (known) matrix of coefficients, and $\alpha, \beta$ are constant in $\mathbb{R}$. Moreover, $d x+\beta \neq 0$ for all $x \in X$.

To solve LFP problem (1), usually, the method of Charnes and Cooper [4] is used. Let $t=1 /(d x+\beta)$ and $y=x t$; therefore, problem (1) can be converted to the following problem:

$$
\begin{array}{cl}
\text { Min } & f(x)=c y+\alpha t, \\
\text { s.t. } & d y+\beta t=1, \\
& A y-b t \leq 0, \\
& y \geq 0, t \geq 0 .
\end{array}
$$

Theorem 1. Let $\left(y^{*}, t^{*}\right)$ be an optimal solution of problem (2); then, $x^{*}=y^{*} / t^{*}$ is an optimal solution of LFP problem (1).

Proof. See Charnes and Cooper [4].

2.2. Multiobjective Optimization. Consider the following Multiobjective programming problem:

$$
\begin{array}{ll}
\text { Min } & f(x)=\left(f_{1}(x), \ldots, f_{p}(x)\right)^{t}, \\
\text { s.t. } & x \in X,
\end{array}
$$


where $X \subseteq \mathbf{R}^{n}$ is the feasible set and $f_{i}(x), i=1,2, \ldots, p$, are the objective functions. Usually, there exist conflicts among objective functions in their targets; then, usually, there does not exist any feasible solution of an MOP problem that optimizes all objective functions. Therefore, the notions of efficient solutions and weakly efficient solutions are introduced in MOP instead of optimal solutions.

Definition 2. A point $\bar{x} \in X$ is an efficient solution or paretooptimal solution of problem (3) if there is no other $x \in X$ such that $f_{k}(x) \leq f_{k}(\bar{x})$ for all $k=1, \ldots, p$ and $f_{j}(x)<f_{j}(\bar{x})$ for at least one $j \in\{1, \ldots, p\}$.

Definition 3. A point $\bar{x} \in X$ is a weakly efficient solution of problem (3) if there is no other $x \in X$ such that $f_{k}(x)<f_{k}(\bar{x})$ for all $k=1, \ldots, p$.

Definition 4. A point $\bar{x} \in X$ is a strictly efficient solution of problem (3) if there is no other $x \in X, x \neq \bar{x}$, such that $f_{k}(x) \leq f_{k}(\bar{x})$ for all $k=1, \ldots, p$.

Remark 5. Definitions 2,3 , and 4 imply that each strictly efficient solution is an efficient solution and each efficient solution is a weakly efficient solution but the reverses, necessarily, are not true.

There are many methods to find the efficient solutions in MOP. One of the most well-known methods to identify the efficiency status of a feasible solution was proposed by Benson [1] which is presented here in synopsis.

The idea and the aim of Benson's method are on identifying the efficiency status of a feasible solution of MOP. If the mentioned feasible solution is not efficient and then Benson's method finds an efficient solution of the MOP which dominates the mentioned inefficient feasible solution. This found efficient solution is called the projection of the inefficient solution on the efficient space. The problem proposed by Benson [1] maximizes the distance between the vector of objective functions corresponding to the under-assessment feasible solution $\left(x^{0}\right)$ and the vectors of objective functions corresponding to all feasible solutions which dominate $f\left(x^{0}\right)$. The distance function in Benson's method is $\|\cdot\|_{1}\left(L^{1}\right.$-norm).

By the above explanation, Benson's method is modeled as follows:

$$
\begin{array}{ll}
\operatorname{Max} & \sum_{k=1}^{p} l_{k}, \\
\text { s.t. } & f_{k}(x)+l_{k} \leq f_{k}\left(x^{0}\right), \quad k=1,2, \ldots, p, \\
& l_{k} \geq 0, \\
& x \in X .
\end{array}
$$

Theorem 6. If $x^{*}$ is an optimal solution of problem (4), then $x^{*}$ is an efficient solution of problem (3).

Proof. See Benson [1].

Theorem 7. If the optimal objective value of (4) equals zero, then the feasible solution $x^{0} \in X$ is efficient.
Proof. See Benson [1].

Another technique to solve the multiobjective optimization problems is the $\varepsilon$-constraint method which was introduced by Haimes et al. [2]. In this method, only one of the original objectives is minimized, while the others are transformed to constraints. The $\varepsilon$-constraint problem associated with the MOP problem (3) is formulated as

$$
\begin{array}{ll}
\text { Min } & f_{j}(x), \\
\text { s.t. } & f_{k}(x) \leq \varepsilon_{k}, \quad k=1,2, \ldots, p, k \neq j, \\
& x \in X,
\end{array}
$$

where $\varepsilon \in R^{p}$.

Theorem 8. Let $x^{*}$ be an optimal solution of (5) for some $j$, and then $x^{*}$ is a weakly efficient solution of (3).

Proof. See Haimes et al. [2].

Theorem 9. Let $x^{*}$ be a unique optimal solution of (5) for some $j$, then $x^{*}$ is a strictly efficient solution of (3), and therefore $x^{*}$ is efficient.

Proof. See Haimes et al. [2].

\section{The Proposed Approaches}

The multiobjective linear fractional programming problems are formulated as follows:

$$
\begin{aligned}
& \text { Min } f(x)=\left(\frac{c_{1} x+\alpha_{1}}{d_{1} x+\beta_{1}}, \ldots, \frac{c_{p} x+\alpha_{p}}{d_{p} x+\beta_{p}}\right)^{t}, \\
& \text { s.t. } \quad x \in X=\left\{x \in R^{n}: A x \leq b, x \geq 0\right\},
\end{aligned}
$$

where $X$ is a nonempty and bounded set, $A \in R^{m \times n}, b \in R^{m}$, $c_{k}, d_{k} \in R^{n}$, and $\alpha_{k}, \beta_{k} \in R$ for all $k=1,2, \ldots, p$. Moreover, the feasible set $X$ is a polytope (bounded polyhedron) and $d_{k} x+\beta_{k}$ is positive or negative in all feasible solutions of MOLFP problem (6) for all $k=1,2, \ldots, p$. Without loss of generality, in this paper we assume that $d_{k} x+\beta_{k}>0$ for all $x \in X$ and $k=1,2, \ldots, p$.

To explain the proposed approaches, at first, we express Benson's method for MOLFP problem (6) and convert it to a linear programming problem. Benson's problem corresponding to MOLFP problem (6) is as follows:

$$
\begin{array}{ll}
\operatorname{Max} & \sum_{k=1}^{p} l_{k}, \\
\text { s.t. } & \frac{c_{k} x+\alpha_{k}}{d_{k} x+\beta_{k}}+l_{k} \leq f_{k}\left(x^{0}\right), \quad k=1,2, \ldots, p, \\
& l_{k} \geq 0, \quad k=1,2, \ldots, p, \\
& x \in X,
\end{array}
$$

where $x^{0} \in X$ is a feasible solution of (6) and $f_{k}\left(x^{0}\right)=\left(c_{k} x^{0}+\right.$ $\left.\alpha_{k}\right) /\left(d_{k} x^{0}+\beta_{k}\right), k=1, \ldots, p$. 
Let $X \neq \emptyset$ be a bounded set. It implies that the feasible set of (7) is bounded. Then, the efficient MOP problem (6) is not empty and problem (7) has an optimal solution.

Regarding the nonlinearity of the first group of the constraints of problem (7), we convert these constraints to linear constraints as follows:

$$
\begin{aligned}
\frac{c_{k} x+\alpha_{k}}{d_{k} x+\beta_{k}}+l_{k} & \leq f_{k}\left(x^{0}\right) \Longrightarrow \\
\frac{c_{k} x+\alpha_{k}}{d_{k} x+\beta_{k}}-f_{k}\left(x^{0}\right) & \leq-l_{k} \Longrightarrow \\
\frac{c_{k} x+\alpha_{k}-f_{k}\left(x^{0}\right)\left(d_{k} x+\beta_{k}\right)}{d_{k} x+\beta_{k}} & \leq-l_{k},
\end{aligned}
$$

for $k=1,2, \ldots, p$. Since, for all $x \in X$, we have $d_{k} x+\beta_{k}>0$ and $-l_{k} \leq 0, k=1,2, \ldots, p$, we have $c_{k} x+\alpha_{k}-f_{k}\left(x^{0}\right)\left(d_{k} x+\right.$ $\left.\beta_{k}\right) \leq 0$ for $k=1,2, \ldots, p$.

Consider the following linear programming problem:

$$
\begin{array}{ll}
\operatorname{Max} & \sum_{k=1}^{p} l_{k}^{\prime}, \\
\text { s.t. } & c_{k} x+\alpha_{k}+l_{k}^{\prime} \leq f_{k}\left(x^{0}\right)\left(d_{k} x+\beta_{k}\right), \\
& k=1,2, \ldots, p, \\
& l_{k}^{\prime} \geq 0, \quad k=1,2, \ldots, p, \\
& x \in X .
\end{array}
$$

The following theorem shows the relation between the optimal objective value of the above problem and the efficient solutions of MOLFP problem (6).

Theorem 10. If the optimal objective value of (9) equals zero, then the feasible solution $x^{0} \in X$ is an efficient solution of MOLFP problem (6).

Proof. With respect to the constraints of problem (9) and because of $l_{k}^{\prime} \geq 0, k=1,2, \ldots, p$, we conclude the following results for all $x$ in the feasible set of (9):

$$
\begin{aligned}
& c_{k} x+\alpha_{k}+l_{k}^{\prime} \leq f_{k}\left(x^{0}\right)\left(d_{k} x+\beta_{k}\right) \Longrightarrow \\
& c_{k} x+\alpha_{k} \leq f_{k}\left(x^{0}\right)\left(d_{k} x+\beta_{k}\right), \\
& \quad k=1,2, \ldots, p .
\end{aligned}
$$

Because of $d_{k} x+\beta_{k}>0$ for all $x \in X$, we have $\left(c_{k} x+\alpha_{k}\right) /\left(d_{k} x+\right.$ $\left.\beta_{k}\right) \leq f_{k}\left(x^{0}\right)$ for all $k=1,2, \ldots, p$.

Since all coefficients of variables $l_{k}^{\prime}, k=1,2, \ldots, p$, in the objective function of problem (9) are positive, they are equal to one, and $x^{*}$ is an optimal solution of (9); therefore, $l_{k}^{\prime}$ associated with $x^{*}$, which is shown by $l_{k}^{\prime *}$, satisfies in the following relations:

$$
\begin{aligned}
& c_{k} x^{*}+\alpha_{k}+l_{k}^{\prime *}=f_{k}\left(x^{0}\right)\left(d_{k} x^{*}+\beta_{k}\right) \Longrightarrow \\
&-l_{k}^{\prime *}=c_{k} x^{*}+\alpha_{k}-f_{k}\left(x^{0}\right)\left(d_{k} x^{*}+\beta_{k}\right), \\
& k=1,2, \ldots, p,
\end{aligned}
$$

and so

$$
-\sum_{k=1}^{p} l_{k}^{\prime *}=\sum_{k=1}^{p}\left(c_{k} x^{*}+\alpha_{k}-f_{k}\left(x^{0}\right)\left(d_{k} x^{*}+\beta_{k}\right)\right) .
$$

Now, suppose that $\left(x^{*}, l^{\prime *}\right)=\left(x^{*}, 0\right)$ is an optimal solution of (9) and by contradiction suppose that $x^{0}$ is not an efficient solution of MOLFP problem (6). Then, there exists a feasible solution $\bar{x} \in X$ such that $\left(c_{k} \bar{x}+\alpha_{k}\right) /\left(d_{k} \bar{x}+\beta_{k}\right) \leq$ $\left(c_{k} x^{0}+\alpha_{k}\right) /\left(d_{k} x^{0}+\beta_{k}\right)=f_{k}\left(x^{0}\right)$ for all $k$ and $\left(c_{j} \bar{x}+\alpha_{j}\right) /\left(d_{j} \bar{x}+\right.$ $\left.\beta_{j}\right)<\left(c_{j} x^{0}+\alpha_{j}\right) /\left(d_{j} x^{0}+\beta_{j}\right)=f_{j}\left(x^{0}\right)$ for some $j$.

Let $I=\left\{k \mid\left(c_{k} \bar{x}+\alpha_{k}\right) /\left(d_{k} \bar{x}+\beta_{k}\right)=f_{k}\left(x^{0}\right)\right\}$ and $\bar{l}_{k}^{\prime}=$ $f_{k}\left(x^{0}\right)\left(d_{k} \bar{x}+\beta_{k}\right)-\left(c_{k} \bar{x}+\alpha_{k}\right)$ for all $k=1,2, \ldots, p$. These relations imply that $\bar{l}_{k}^{\prime}=0$ for all $k \in I$ and $\bar{l}_{k}^{\prime}>0$ for all $k \notin I$. Because of $\bar{l}_{k}^{\prime} \geq 0, k=1,2, \ldots, p$, we conclude that $\left(\bar{x}, \bar{l}^{\prime}\right)$ is a feasible solution of (9).

Also, $\{1,2, \ldots, p\} \backslash I \neq \emptyset$ and we have $\sum_{k=1}^{p} \bar{l}_{k}^{\prime}>0$ which contradicts with the assumption of the optimality of $\left(x^{*}, l^{*}\right)=\left(x^{*}, 0\right)$ in $(9)$. Because the objective value of $(9)$ corresponding to $\left(\bar{x}, \bar{l}^{\prime}\right)$ is strictly positive while the objective value of (9) corresponding to $\left(x^{*}, l^{\prime *}\right)$ is zero, therefore $x^{*}$ is an efficient solution of (6).

Now, we express the $\varepsilon$-constraint method for MOLFP problem (6). Let $x^{0}$ be a feasible solution of (6) and $\varepsilon_{k}=$ $f_{k}\left(x^{0}\right), k=1,2, \ldots, p$. Therefore, $\varepsilon$-constraint problem (5) corresponding to MOLFP problem (6) is as follows:

$$
\begin{array}{ll}
\operatorname{Min} & \frac{c_{j} x+\alpha_{j}}{d_{j} x+\beta_{j}}, \\
\text { s.t. } & A x \leq b, \\
& \frac{c_{k} x+\alpha_{k}}{d_{k} x+\beta_{k}} \leq f_{k}\left(x^{0}\right), \quad k=1, \ldots, p, k \neq j, \\
& x \geq 0 .
\end{array}
$$

We transform the above nonlinear programming problem to a linear programming problem using Charnes and Cooper's transformation method. Let $t=1 /\left(d_{j} x+\beta_{j}\right)$ and $y=x t$. The above problem is transformed to the following linear programming problem:

$$
\begin{array}{ll}
\text { Min } & c_{j} y+\alpha_{j} t, \\
\text { s.t. } & A y-b t \leq 0, \\
& c_{k} y+\alpha_{k} t \leq f_{k}\left(x^{0}\right)\left(d_{k} y+\beta_{k} t\right), \\
& k=1, \ldots, p, k \neq j, \\
& d_{j} y+\beta_{j} t=1, \\
& y \geq 0, t \geq 0 .
\end{array}
$$

Theorem 11. Let $\left(y^{*}, t^{*}\right)$ be an optimal solution of problem (14) for some $j$, and then $x^{*}=y^{*} / t^{*}$ is an optimal solution of problem (13). 
Proof. At first, we claim that $t$ is not zero in all feasible solutions of (14). By contradiction, suppose that $(y, t)=(\bar{y}, 0)$ is a feasible solution of (14). So $A \bar{y} \leq 0$ and $\bar{y} \geq 0$. These imply that $\bar{y}$ is a recession direction of $X$ (the feasible set of MOLFP (6)) which is a contradiction to the primary assumption of the boundedness of $X$. So $t>0$ in all feasible solutions, and in particular $t$ is positive in all optimal solutions of (14).

$\left(y^{*}, t^{*}\right)$ is an optimal solution of $(14)$ and $t^{*}>0$. Let $x^{*}=$ $y^{*} / t^{*}$. We prove that $x^{*}$ is an optimal solution of (13).

$$
\begin{aligned}
A x^{*} t^{*} & =A y^{*} \leq b t^{*} \Longrightarrow \\
A x^{*} & \leq b .
\end{aligned}
$$

Moreover,

$$
\begin{aligned}
c_{k} x^{*} t^{*}+\alpha_{k} t^{*} & =c_{k} y^{*}+\alpha_{k} t^{*} \leq f_{k}\left(x^{0}\right)\left(d_{k} y^{*}+\beta_{k} t^{*}\right) \\
& =f_{k}\left(x^{0}\right)\left(d_{k} x^{*} t^{*}+\beta_{k} t^{*}\right),
\end{aligned}
$$

for all $k=1,2, \ldots, p$ and $k \neq j$. These imply that

$$
\frac{c_{k} x^{*}+\alpha_{k}}{d_{k} x^{*}+\beta_{k}} \leq f_{k}\left(x^{0}\right), \quad k=1, \ldots, p, k \neq j .
$$

So $x^{*}$ is a feasible solution of problem (13). We show that $x^{*}$ is an optimal solution of (13). By contradiction, suppose that $x^{*}$ is not an optimal solution of (13). Therefore, there exists feasible solution $\bar{x}$ of (13) such that

$$
\frac{c_{j} \bar{x}+\alpha_{j}}{d_{j} \bar{x}+\beta_{j}}<\frac{c_{j} x^{*}+\alpha_{j}}{d_{j} x^{*}+\beta_{j}} .
$$

Let $\bar{t}=1 /\left(d_{j} \bar{x}+\beta_{j}\right)$ and $\bar{y}=\bar{x} \bar{t}$. It is easy to see that $(\bar{y}, \bar{t})$ is a feasible solution of (14). Moreover, from (18) we have $c_{j} \bar{y}+$ $\alpha_{j} \bar{t}<c_{j} y^{*}+\alpha_{j} t^{*}$, which contradicts with the assumption that $\left(y^{*}, t^{*}\right)$ is an optimal solution of (14). Therefore $x^{*}=$ $y^{*} / t^{*}$ is an optimal solution of problem (13), and the proof is completed.

Theorem 12. Let $\left(y^{*}, t^{*}\right)$ be an optimal solution of problem (14) for some $j$, and then $x^{*}=y^{*} / t^{*}$ is a weakly efficient solution for MOLFP problem (6). In particular, if $\left(y^{*}, t^{*}\right)$ is a unique optimal solution of (14), then $x^{*}=y^{*} / t^{*}$ is a strictly efficient solution and an efficient solution for MOLFP problem (6).

Proof. Suppose that $\left(y^{*}, t^{*}\right)$ is an optimal solution of (14). Therefore, by Theorem 11, $x^{*}=y^{*} / t^{*}$ is an optimal solution of (13) and, regarding to Theorem $8, x^{*}$ is weakly efficient solution of MOLFP problem (6). Moreover, if $\left(y^{*}, t^{*}\right)$ is a unique optimal solution of problem (14), then $x^{*}=y^{*} / t^{*}$ is a unique optimal solution of problem (13) and, regarding to Theorem $9, x^{*}$ is a strictly efficient solution and, therefore, it is an efficient solution of MOLFP problem (6), which completes the proof.

Remark 13. By Theorem 12, we conclude that an optimal solution of problem (14) clarifies a weakly efficient solution of MOLFP problem (6). If the optimal solution of (14) is unique, then this optimal solution determines an efficient solution of MOLFP problem (14). Otherwise, if problem (14) has alternative optimal solutions, then to clarify the efficiency status of an optimal solution of (14) we use problem (9) and Theorem 10 .

\section{Examples}

Example 1. Consider the following MOLFP problem:

$$
\begin{array}{ll}
\operatorname{Min} & f_{1}(x)=\frac{x_{1}+2 x_{2}+5}{x_{1}+x_{2}+2}, \\
\text { Min } & f_{2}(x)=\frac{x_{1}+x_{2}+10}{-x_{1}+2 x_{2}+4}, \\
\text { s.t. } & x_{1} \leq 3, \\
& x_{2} \leq 4, \\
& x_{1}, x_{2} \geq 0 .
\end{array}
$$

According to problem (14) for $j=1$, problem (19) can be converted to the following problem:

$$
\begin{array}{ll}
\operatorname{Min} & y_{1}+2 y_{2}+5 t \\
\text { s.t. } & y_{1}+y_{2}+10 t \leq f_{2}\left(x^{0}\right)\left(-y_{1}+2 y_{2}+4 t\right) \\
& y_{1}+y_{2}+2 t=1 \\
& y_{1}-3 t \leq 0 \\
& y_{2}-4 t \leq 0 \\
& y_{1}, y_{2}, t \geq 0
\end{array}
$$

where $x^{0}$ is a feasible solution of problem (19).

For each arbitrary feasible point, which is called an underassessment feasible solution, we will solve problem (20) and obtain the projection of the under-assessment feasible solution of problem (19) on the weakly efficient space of that. Then, we check the efficient status of the projection solution using problem (9) and Theorem 10. The optimal solutions of problem (20) corresponding to several under-assessment feasible solutions and their efficiency statuses have been listed in Table 1. Note that if $\left(y^{*}, t^{*}\right)$ is a unique optimal solution of problem (20), then $y^{*} / t^{*}$ is an efficient solution for MOLFP problem (19) and we do not need to check the projection by (9). Figure 1 shows the feasible set and the efficient solutions of (19). The rectangle with vertex $A, B, C, O$ and its interior is the set of feasible solutions. All points on bold segments $A B$ and $B C$ are the efficient solutions of (19).

Note that points $A, B$, and $C$ are extremely efficient. If we choose them as under-assessment points $\left(x^{0}\right)$ and solve problem (20), then the optimal solutions of (20) corresponding to the three points, which are the projections of these feasible solutions on the efficient space, are unique and equal to themselves.

Table 1 presents the results of examining 7 feasible solutions of (19) of which 6 feasible solutions are on the boundary 
TABLE 1: Results of Example 1 using different feasible solutions of (19).

\begin{tabular}{lccc}
\hline Row & $\begin{array}{c}\text { Feasible solution } \\
\left(x^{0}\right)\end{array}$ & $\begin{array}{c}\text { Efficiency status } \\
\text { of } x^{0}\end{array}$ & Projection \\
\hline 1 & $A=(0.0000,4.0000)$ & Efficient & $(0.0000,4.0000)$ \\
2 & $B=(3.0000,4.0000)$ & Efficient & $(3.0000,4.0000)$ \\
3 & $C=(3.0000,0.0000)$ & Efficient & $(3.0000,0.0000)$ \\
4 & $(2.1078,4.0000)$ & Efficient & $(2.1078,4.0000)$ \\
5 & $(0.0000,0.0000)$ & Inefficient & $(3.0000,2.6250)$ \\
6 & $(0.0000,2.0000)$ & Inefficient & $(1.6000,4.0000)$ \\
7 & $(1.6789,1.5678)$ & Inefficient & $(3.0000,2.7424)$ \\
\hline
\end{tabular}

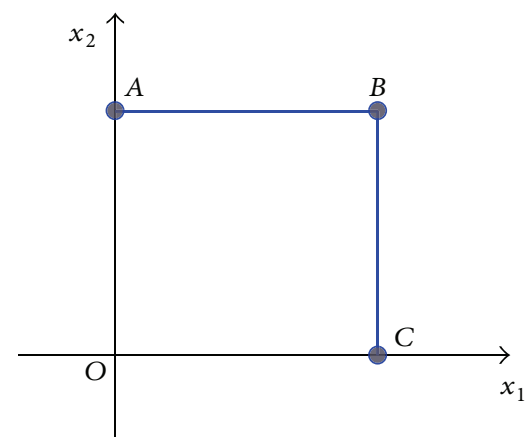

$$
\begin{aligned}
& A=(0,4) \\
& B=(3,4) \\
& C=(3,0)
\end{aligned}
$$

FIGURE 1: Feasible and efficient sets of problem (19).

of the feasible set and one of them is an interior point of the feasible set. The projection points corresponding to the feasible points in rows 1 to 4 are themselves and they are efficient by problem (9) which are also endorsed by Figure 1. In particular, the points in rows 1 to 3 are basic feasible solutions of (19) and so they are the efficient extreme points of (19). Also the feasible solution in row 4 is on segments $A B$.

The other feasible solutions in rows 5 to 7 are inefficient. The points in rows 5 and 6 are on the inefficient boundary of the feasible set of (19) and their projections are on segments $B C$ and $A B$, respectively. The point in row 7 is an interior point of the feasible set of (19) and its projection is on segment $B C$.

Example 2. Here, we consider an MOLFP problem with three objective functions which has been extracted from Kornbluth and Steuer [10] as follows:

$$
\begin{array}{ll}
\text { Min } & f_{1}(x)=\frac{-x_{1}+4}{-x_{2}+3}, \\
\text { Min } & f_{2}(x)=\frac{x_{1}-4}{x_{2}+1}, \\
\text { Min } & f_{3}(x)=x_{1}-x_{2}, \\
\text { s.t. } & -x_{1}+4 x_{2} \leq 0, \\
& x_{1}-0.5 x_{2} \leq 4, \\
& x_{1}, x_{2} \geq 0 .
\end{array}
$$

TABLE 2: The projections of several feasible solutions of (21) and their efficiency statuses.

\begin{tabular}{lccc}
\hline Row & Feasible point $\left(x^{0}\right)$ & $\begin{array}{c}\text { Efficiency status of } \\
x^{0}\end{array}$ & Projection \\
\hline 1 & $A=(0.0000,0.0000)$ & Efficient & $(0.0000,0.0000)$ \\
2 & $C=(4.5714,1.1429)$ & Efficient & $(4.5714,1.1429)$ \\
3 & $(4.0000,1.0000)$ & Efficient & $(4.0000,1.0000)$ \\
4 & $(1.5558,0.0000)$ & Efficient & $(1.5558,0.0000)$ \\
5 & $B=(4.0000,0.0000)$ & Inefficient & $(4.0000,0.0706)$ \\
& & (Weakly efficient) & \\
6 & $D=(1.3000,0.3000)$ & Inefficient & $($ Weakly efficient) \\
7 & $(0.5000,0.0625)$ & Inefficient & $(0.4255,0.00000)$ \\
8 & $(0.7690,0.1168)$ & Inefficient & $(0.6381,0.0000)$ \\
\hline
\end{tabular}

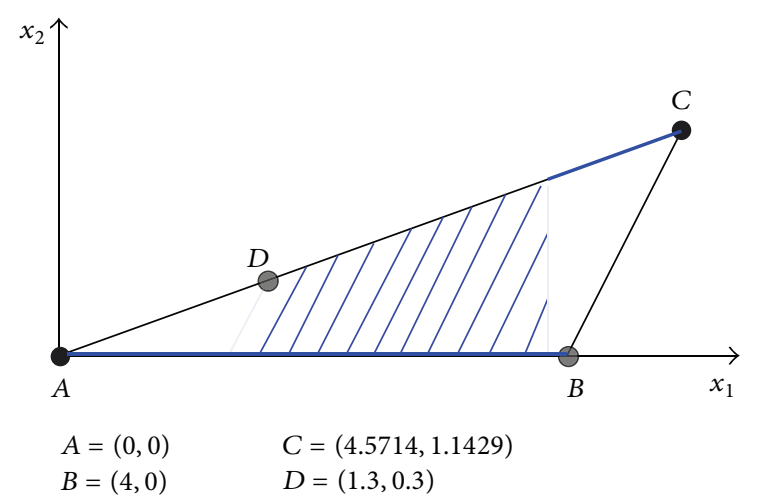

FIGURE 2: Feasible and efficient regions of problem (21).

The feasible set of the above MOLFP problem has been shown in Figure 2. The feasible set is a triangle with vertices $A, B$, and $C$. All the bold segments are efficient except points $B$ and $D$ which are weakly efficient. The other points in the feasible set are inefficient. For more illustration, see Kornbluth and Steuer [10].

Regarding (6), (13), and (14) for $j=2$, MOLFP problem (21) is transformed to the following linear programming problem:

$$
\begin{array}{ll}
\text { Min } & y_{1}-4 t, \\
\text { s.t. } & -y_{1}+4 t \leq f_{1}\left(x^{0}\right)\left(-y_{2}+3 t\right), \\
& y_{1}-y_{2} \leq f_{3}\left(x^{0}\right) t, \\
& y_{2}+t=1 \\
& -y_{1}+4 y_{2} \leq 0 \\
& y_{1}-0.5 y_{2}-4 t \leq 0 \\
& y_{1}, y_{2}, t \geq 0
\end{array}
$$

where $x^{0}$ is a feasible solution of problem (21).

By solving linear programming problem (22) for each arbitrary feasible solution of (21), we can obtain a projection of $x^{0}$ on the weakly efficient space of (21). The results of solving problem (21) for several feasible solutions have been listed in Table 2. In Table 2, we see that columns 2 and 4 
corresponding to rows 1 to 4 are the same. In other words, the projections of the under-assessment feasible solutions in rows 1 to 4 are themselves and they are efficient using problem (9) corresponding to (21). Furthermore, all other feasible solutions in rows 5 to 8 are inefficient and their projections, obtained from (22), are efficient.

\section{Conclusion}

In real world, many of events and problems are modeled as multiobjective programming problems. Multiobjective linear fractional programming (MOLFP) problems have special structures among multiobjective programming problems. The feasible sets of them are polyhedron and also their objective functions are fractional whose nominators and denominators are affine functions.

Because of the special structure of the MOLFP problems, in this paper we suggest a linear programming technique to find the efficiency status of a feasible solution of an MOLFP problem, and if it is not efficient, then we project it on the efficient space of the MOLFP problem. For this purpose, we propose two linear programming problems. One of the proposed linear programming problems attempts to find a feasible solution of the MOLFP problem which dominates the under-assessment feasible solution. If there does not exist any optimal solution of the proposed linear programming problem which equals the under-assessment feasible solution, then the under-assessment feasible solution is inefficient. In this case the optimal solution is a projection of the underassessment feasible solution on the weakly efficient set of the MOLFP problems. Otherwise, if the optimal solution of the proposed problem is the same as the under-assessment feasible solution, then the feasible solution is weakly efficient. Another proposed linear programming problem identifies the efficiency statuses of the feasible solutions. We can check the efficiency status of an arbitrary feasible solution or a projection point, obtained by another problem, by this linear programming problem.

\section{Competing Interests}

The authors declare that there are no competing interests regarding the publication of this paper.

\section{References}

[1] H. P. Benson, "Existence of efficient solutions for vector maximization problems," Journal of Optimization Theory and Applications, vol. 26, no. 4, pp. 569-580, 1978.

[2] Y. Y. Haimes, L. S. Lasdon, and D. A. Wismer, "On a bicriterion formulation of the problems of integrated system identification and system optimization," IEEE Transactions on Systems, Man, and Cybernetics, vol. 1, pp. 296-297, 1971.

[3] V. Chankong and Y. Haimes, Multiobjective Decision Making: Theory and Methodology, Elsevier Science, New York, NY, USA, 1983.

[4] A. Charnes and W. W. Cooper, "Programming with linear fractional functionals," Naval Research Logistics, vol. 9, pp. 181$186,1962$.
[5] P. C. Gilmore and R. E. Gomory, "A linear programming approach to the cutting stock problem. Part II," Operations Research, vol. 11, no. 6, pp. 863-888, 1963.

[6] B. Martos, "Hyperbolic programming," Naval Research Logistics, vol. 11, pp. 135-155, 1964.

[7] T. Illés, Á. Szirmai, and T. Terlaky, "The finite criss-cross method for hyperbolic programming," European Journal of Operational Research, vol. 114, no. 1, pp. 198-214, 1999.

[8] S. F. Tantawy, "A new procedure for solving linear fractional programming problems," Mathematical and Computer Modelling, vol. 48, no. 5-6, pp. 969-973, 2008.

[9] A. O. Odior, "An approach for solving linear fractional programming problems," International Journal of Engineering \& Technology, vol. 1, pp. 298-304, 2012.

[10] J. S. H. Kornbluth and R. E. Steuer, "Multiple objective linear fractional programming," Management Sciences, vol. 27, no. 9, pp. 1024-1039, 1981.

[11] B. Metev and D. Gueorguieva, "A simple method for obtaining weakly efficient points in multiobjective linear fractional programming problems," European Journal of Operational Research, vol. 126, no. 2, pp. 386-390, 2000.

[12] R. Caballero and M. Hernández, "The controlled estimation method in the multiobjective linear fractional problem," Computers \& Operations Research, vol. 31, no. 11, pp. 1821-1832, 2004.

[13] W. Dinkelbach, "On nonlinear fractional programming," Management Science, vol. 13, pp. 492-498, 1967.

[14] Y. Almogy and O. Levin, "A class of fractional programming problems," Operations Research, vol. 19, pp. 57-67, 1971.

[15] J.-P. Crouzeix, J. A. Ferland, and S. Schaible, "An algorithm for generalized fractional programs," Journal of Optimization Theory and Applications, vol. 47, no. 1, pp. 35-49, 1985.

[16] J. E. Falk and S. W. Palocsay, "Image space analysis of generalized fractional programs," Journal of Global Optimization, vol. 4, no. 1, pp. 63-88, 1994.

[17] K. Tammer, C. Tammer, and E. Ohlenderf, "Multicriterial fractional optimization," in Parametric Optimization and Related Topics IV, J. Guddat, H. T. Jongen, F. Nozicka, G. Still, and F. Twilt, Eds., pp. 359-370, Peter Lang, Berlin, Germany, 1997.

[18] C. C. Skiscim and S. W. Palocsay, "Minimum spanning trees with sums of ratios," Journal of Global Optimization, vol. 19, no. 2, pp. 103-120, 2001.

[19] S. Schaible and J. Shi, "Recent developments in fractional programming: single ratio and maxmin case," in Proceedings of the 3 rd International Conference in Nonlinear Analysis, W. Takahashi and T. Tanaka, Eds., pp. 493-506, Yokohama Publisher, Yokohama, Japan, 2004.

[20] J. P. Costa, "Computing non-dominated solutions in MOLFP," European Journal of Operational Research, vol. 181, no. 3, pp. 1464-1475, 2007.

[21] T. Kuno, "A branch-and-bound algorithm for maximizing the sum of several linear ratios," Journal of Global Optimization, vol. 22, no. 1-4, pp. 155-174, 2002.

[22] N. T. H. Phuong and H. Tuy, "A unified monotonic approach to generalized linear fractional programming," Journal of Global Optimization, vol. 26, no. 3, pp. 229-259, 2003.

[23] Y. Dai, J. Shi, and S. Wang, "Conical partition algorithm for maximizing the sum of DC ratios," Journal of Global Optimization, vol. 31, no. 2, pp. 253-270, 2005.

[24] J. P. Costa and J. M. Alves, "A reference point technique to compute nondominated solutions in MOLFP," Journal of Mathematical Sciences, vol. 161, no. 6, pp. 820-831, 2009. 
[25] J. Matejaš and T. Perić, "A new iterative method for solving multiobjective linear programming problem," Applied Mathematics and Computation, vol. 243, pp. 746-754, 2014.

[26] E. Valipour, M. A. Yaghoobi, and M. Mashinchi, "An iterative approach to solve multiobjective linear fractional programming problems," Applied Mathematical Modelling, vol. 38, no. 1, pp. 38-49, 2014. 


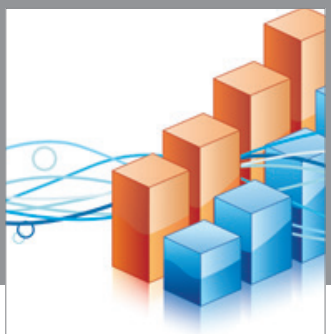

Advances in

Operations Research

vatem alat4

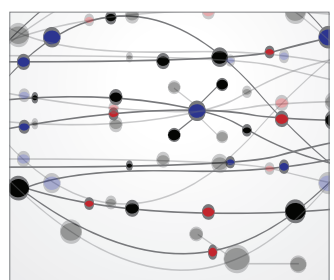

\section{The Scientific} World Journal
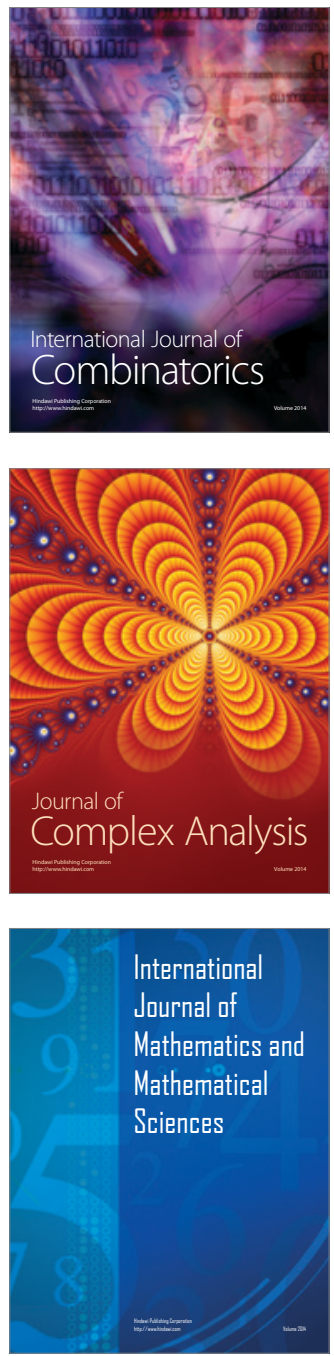
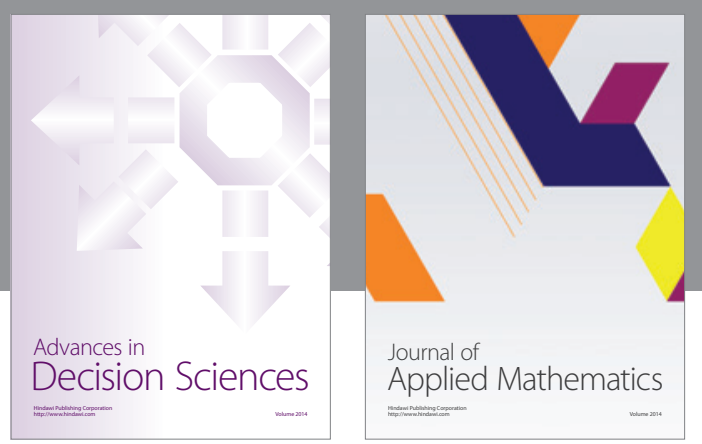

Algebra

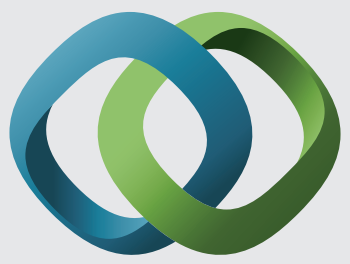

\section{Hindawi}

Submit your manuscripts at

http://www.hindawi.com
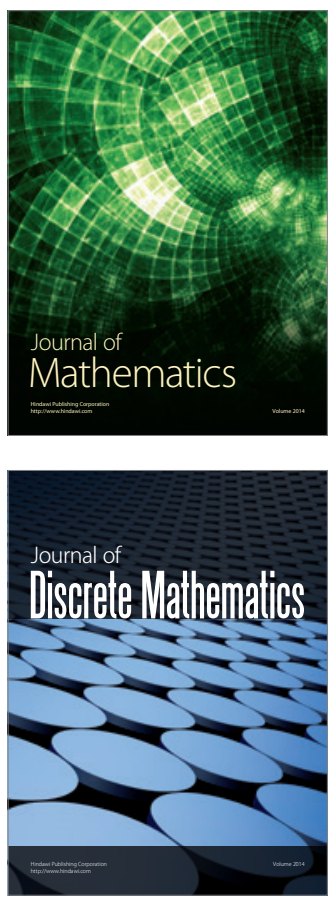

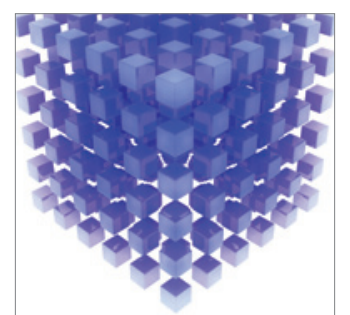

Mathematical Problems in Engineering
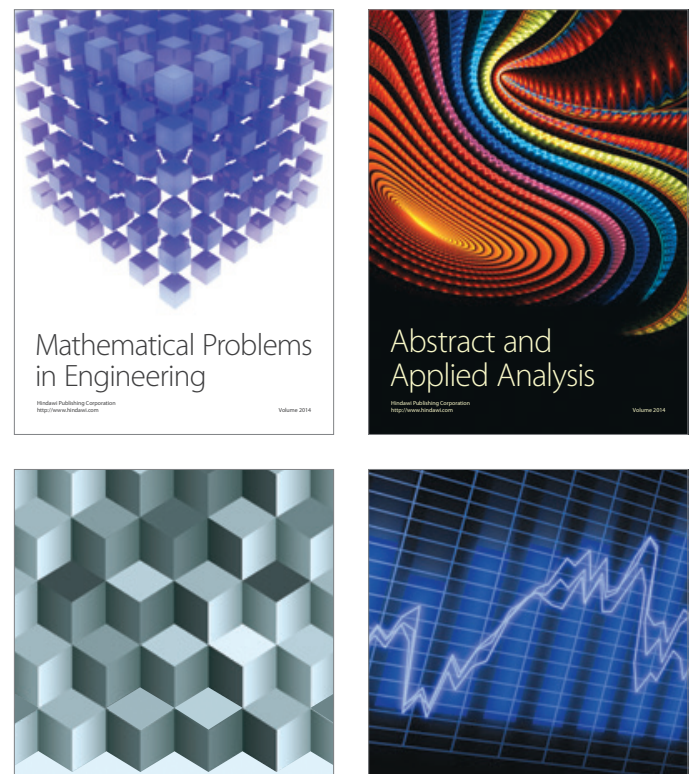

Journal of

Function Spaces

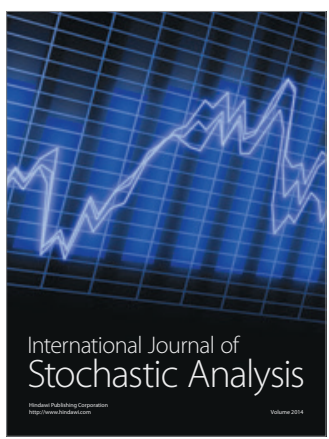

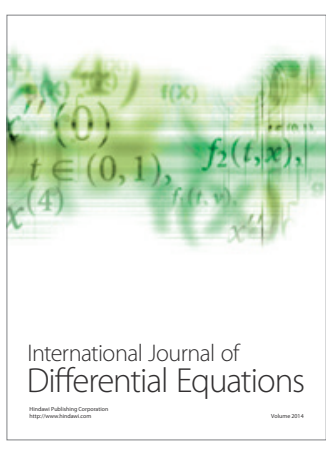
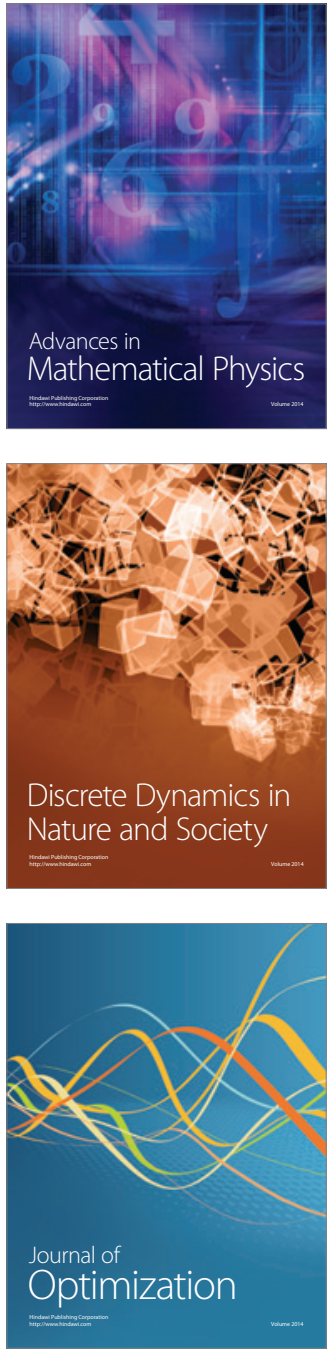